\title{
PROPHYLACTIC VACCINES AGAINST CANCERS OF NON-INFECTIOUS ORIGIN: A DREAM OR A REAL POSSIBILITY?
}

\author{
Vladimír Vonka', Ivan Hirsch ${ }^{2,3}$ \\ ${ }^{1}$ Institute of Haematology and Blood Transfusion, Emeritus, Prague, Czech Republic \\ ${ }^{2}$ Department of Genetics and Microbiology, Faculty of Science, Charles University, BIOCEV, Vestec, Czech Republic \\ ${ }^{3}$ Institute of Organic Chemistry and Biochemistry, Academy of Sciences of the Czech Republic, Prague, Czech Republic
}

\begin{abstract}
SUMMARY
The dramatic progress in tumour biology and immunology in the past several years has opened new avenues for the treatment and prevention of cancer. One of the great contributions of the immunotherapeutic approaches is an increasing understanding of the immunology of cancer, which is, gradually creating conditions for the development of prophylactic anti-cancer vaccines. Efficient vaccines have been developed and employed for the prophylaxis of two frequent cancers of viral origin, namely cervical cancer and liver cancer. The new knowledge on the interactions between the immune system and the malignant tumors seems to provide means for the development of prophylactic vaccines against cancers developing due to the mutations in the proto-oncogenes converting their products into oncoproteins. According to the present estimates, these cancers form a great majority of human malignancies. Recent evidence has indicated that the immune system recognizes such mutated proteins, and that the development of cancer is due to the failure of the immune system to eliminate neoplastic cells. Followingly, it can be expected that inducing immunity against the mutated epitopes will increase the capacity of the body to deal with the initiated precancerous cells. In the present paper this hypothesis is primarily discussed in the relationship with colorectal cancer (CRC), which seems to be a well-fitting candidate for prophylactic vaccination. CRC is the third most frequent malignancy and the fourth most common cause of cancer mortality. Mutations of two proto-oncogenes, namely RAS and RAF, are involved in the majority of CRC cases and, in addition, they are shared with other human malignancies. Therefore, the strategy to be used for prophylaxis of CRC is discussed together with several other frequent human cancers, namely lung cancer, pancreatic duct cancer and melanoma. The prophylactic vaccines proposed are aimed at the reduction of the incidence of these and, to a lesser extent, some other cancers.
\end{abstract}

Key words: cancer, prophylactic vaccines, proto-oncogenes RAS and RAF

Address for correspondence: V. Vonka, Institute of Haematology and Blood Transfusion, U Nemocnice 2094, Nové Město, 12820 Prague, Czech Republic. E-mail: vvonka@email.cz

https://doi.org/10.21101/cejph.a7219

\section{INTRODUCTION}

\section{The Hypothesis}

In the future, when writing the history of oncology, the first three or four decades of the 21 st century will most likely be termed the era of tumour immunology. In the recent past there has been a considerable progress in our understanding of the immune reactions directed against tumours. The advancement in this field is reflected by an ever-increasing interest in the immunotherapy of cancer (ITC). Medical journals are flooded with papers not only describing its achievements in preclinical models but also in some quite large clinical studies. The still rather small but significant successes convinced government regulatory agencies such as the Food and Drug Administration (FDA) or the European Medicines Agency (EMA) to approve the use of some ITC modalities in clinical practice.

Within the framework of the current enthusiasm for ITC, it is somewhat ignored that the new knowledge concerning the immunology and biology of cancer is offering strong stimuli for the development of new type of prophylactic vaccines. These vaccines have been introduced and are already widely used in the prevention of cancers of viral origin, particularly cervical cancer (1) and liver cancer (2). Efforts to develop prophylactic vaccines against other cancers of infectious origin are under way (3). In other cancers, which make up approximately $80-85 \%$ of the human malignant tumors, no prophylactic vaccines are in health practice at present. However, there is an increasing interest into this problem. In a recent review (4) various strategies of preventative cancer vaccine development were discussed in depth. A lot of attention has been paid to transmembrane glycoprotein mucin 1 (MUC1) protein, which is overexpressed and aberrantly glycosylated in a high number of cancers. The present knowledge of cancer vaccines targeted at the modified MUC1 have recently been summarized in several reviews $(5,6)$. Although MUC1-based vaccines are under consideration for preventative purposes, the present knowledge strongly supports their primary use for therapeutic purposes. Several vaccines based mainly on the so-called cancer testis antigens (CTA) have already been successfully tested on a limited scale for the treatment of patients with precancerous 
lesions $(7,8)$. However, it is difficult to consider such preparations as prophylactic vaccines in their real sense. They seem to stand somewhere between the prophylactic and therapeutic means, exhibiting the properties of both. In the subsequent text the term prophylactic vaccines will be limited to preparations to be used in clinically healthy subjects.

The authors of this paper are convinced that the time has come to seriously consider the development of such preparations. They should be aimed at inducing the immune system to recognize tumour specific antigens at the earliest stage of the oncogenic process and to mount an immune attack against cells harbouring them.

\section{Mutated Proto-oncogenes as Drivers of Oncogenic Process}

The concept proposed in this paper is based on vaccines directed against the mutated proto-oncogenes RAS and RAF considered as frequent drivers of the oncogenic process. We shall mostly be concerned with data submitted by the US National Cancer Institute. The reason for this is obvious. To the best of our knowledge, detailed data on the driving RAS mutations are only available from the USA. For the present proposal we selected cancers which are quite frequent all over the world. This has made us to believe that the cancer vaccines proposed, if effective in the USA, will also suppress a significant proportion of cancers outside the USA.

The respective mutations and the antigenic changes associated with the transformation of normal cells into tumor cells are different not only for different cancers but vary also for cancers developing in the same tissues. CRC or LC can serve as examples (see below). Thus, it is the property of the vaccines proposed that they will not act against all cancers originating in the same tissue; however, on the other hand, they may induce immunity to a wide variety of quite different malignancies sharing the same driving mutation. If our reasoning is correct then to achieve a highly significant suppression of cancer development, not one but several vaccines should gradually be developed and introduced into medical practice on a large scale.

In the study proposed, four cancers will be targeted. The criteria for their selection are as follows: (a) high frequency; (b) sharing the driving mutations with other cancers; (c) an evidence that the mutated oncoproteins are immunogenic; (d) an evidence that the mutations are unequally distributed in the respective cancers, this permitting to use a limited number of vaccines for preventing significant proportion of each of them. For these reasons, the main attention is being paid to colorectal cancer (CRC), lung cancer (LC), pancreatic duct cancer (PDC), and melanoma (MEL). For the present report, the yearly incidence of these cancers was estimated upon data from the Catalogue of Somatic Mutation in Cancer (COSMIC) together with data from the Foundation Medicine database (9). To facilitate the comparison with other databases, both colon and rectal adenocarcinomas were merged for CRC and both lung adenocarcinomas and a squamous cell carcinoma were merged for LC, if not stated otherwise.

\section{RAS and B-RAF Proto-oncogenes}

Proto-oncogenes are components of the cell genome. They play important roles in the cell biology, regulating cell growth and metabolism. Already more than 40 years ago it was revealed that their mutations were involved in the pathogenesis of various forms of cancer. The activated proto-oncogenes are termed cell oncogenes. Since these genes and their oncogenic potential were first discovered as components of oncornavirus genomes, their denotations frequently reflected this fact. Later on, their names were derived from the cancers in which they were discovered, occasionally completed with the initials of the discoverers involved. It is a certain curiosity of the present nomenclature that the oncogene originally found in ASV17 (Avian Sarcoma Virus 17) has been denoted jun. "Junema" means 17 in Japanese.

\section{Occurrence of Carcinogenic Mutations in RAS Gene}

The first oncogene was discovered in the rat sarcoma virus and was denoted RAS $(10,11)$. For decades, RAS oncogene has remained at the focus of interest. This is not surprising because its mutations are involved in the pathogenesis of approximately $25-30 \%$ of human cancers, i.e., more frequently than any other cell oncogene known.

RAS proteins form a family of short proteins with GTPase activity (9-20). Three isoforms of RAS protein exist: Harvey RAS (H-RAS), Kirsten RAS (K-RAS) and neuroblastoma RAS (N-RAS.) Within the RAS family, K-RAS gene is unique in encoding two products due to alternative splicing resulting in divergent C-terminal sequences. These proteins are designated K-RAS4a and K-RAS4b. The four RAS proteins share a high degree of homology in both sequence and structure, but differ in the fourth exon, which encodes the hypervariable region (HVR) responsible for cell membrane targeting. RAS proteins regulate a number of cellular processes such as growth, differentiation, motility, survival, and apoptosis. This is associated with their capability of activating numerous pathways that are of essential importance for the cell behaviour. These include the mitogen activated protein kinase (MAPK) pathway, the constitutive activation of which plays an essential role in the oncogenic process (Fig. 1). With the help of guanine nucleotide exchange factors (GEF), wild RAS proteins cycle between the GDP bound inactive and GTP bound active state. Circulating growth factors stimulate the epidermal growth factor receptor (EGFR). The signal is transmitted to RAS protein, which is activated to GTP bound state capable of activating RAF, the next component of the MAPK cascade. The GTPase activating protein (GAP) stimulates the intrinsic GTPase activity of RAS. The hydrolysis results in the cleavage of GTP-terminal phosphate group converting GTP to GDP. This leads to a temporal switch-off of the physiological signalling of the RAS proteins.

Wild RAS proteins cycle between their active and inactive states and are therefore considered "molecular switches". Mutations in codons 12, 13 and 61 (and rarely in other codons) induce conformational changes rendering down the RAS proteins capability to interact with GAP. This keeps them permanently in an active state, thereby promoting cell proliferation. All four isoforms of mutated RAS share sequence homology in the most critical sites including codons 12, 13 and 61. In spite of this, RAS protein isoforms not only exhibit different functions in normal tissues but also in the malignancies. Evidence has been presented indicating the different effects of the mutated RAS oncoproteins on proliferation, differentiation and resistance to apoptosis. It is likely that the differences in their activities are due to the different 
post-translational modifications of the $\mathrm{C}$-terminus resulting in differences in adherence to plasma membrane but also to membranes of different organelles, such as Golgi apparatus, mitochondria or endoplasmic reticulum.

The central idea of the present report is to propose vaccines capable of inducing immunity to the new epitopes created by the mutations. Because it would not be realistic to prepare effective vaccines to all of them, it seems appropriate to select for the future immunization the most frequent mutations covering significant fractions of the cancers selected. For this purpose, it has been useful to convert the mutant distribution of cases into percentages and then adjust these according to their incidence of the respective malignancies.

According to the Catalogue of Somatic Mutations in Cancer (COSMICv85), of the oncogenic aberrations of the RAS protooncogenes, approximately $75 \%$ are due to the K-RAS gene mutations, approximately $17 \%$ are due to the N-RAS gene mutations, and the remaining $7 \%$ are due to the H-RAS gene mutations (9). Similar though less-comprehensive data have been reported by Cox et al. (21). Data from these two presentations concerning CRC, LC and PDC are shown in Table 1. It is evident that K-RAS mutations have been most frequently detected in PDC and CRC and, to a lesser extent, in LC. Mutations of H-RAS oncogene, although widely employed in preclinical studies, are less frequent in human cancers.

A number of distinct K-RAS mutations have been observed in human cancers (19). The frequency of individual mutants widely differs, and, in addition, the mutations are not uniformly distributed among the cancers selected. This clearly demonstrates that the individual mutations are not created equal (18-27). The incidence of the most frequent K-RAS mutations converted into percentages of either K-RAS mutations detected or all the corresponding cancers tested is shown in Table 2. They indicate that in the triad of CRC, LC and PDC, most of these mutations were located in codon 12 (19). The four K-RAS, mutations selected, namely G12C, G12D, G12V and G13D, were detected in more than $70 \%$ of CRC, LC and PDC with K-RAS mutations. The KRAS G12D mutations were the most frequent, followed by G12V, G12C and G13D mutations. In the case of CRC, LC and PDC, they were detected in approximately 26,14 and 50\%, respectively. K-RAS mutations were rare in MEL.

Although in other studies the percentage distributions of these mutations were somewhat different, sometimes quite markedly, to the best of our knowledge the strong link of K-RAS mutations to CRC, LC and PDC and their most frequent location in codon 12, have never been questioned. The mutations in codons 13 (except G13D) and 61 in these cancers are much less common.

It may be of interest that similar findings were reported by the same authors eight years later (9) and were also obtained by other researchers (24-27). These observations strongly suggest a high stability of molecular profiling of at least some RAS mutated cancers and have turned the corresponding antigens into primary candidates for their including into the future prophylactic vaccines.

Table 1. Percentage distribution of RAS isomer mutations in selected human cancers

\begin{tabular}{|l|c|c|c|c|c|c|c|c|}
\hline \multirow{2}{*}{ Cancer } & \multicolumn{2}{|c|}{ K-RAS } & \multicolumn{2}{c|}{ N-RAS } & \multicolumn{2}{c|}{ H-RAS } & \multicolumn{2}{c|}{ All RAS } \\
\cline { 2 - 11 } & $\mathbf{P}$ & $\mathbf{C}$ & $\mathbf{P}$ & $\mathbf{C}$ & $\mathbf{P}$ & $\mathbf{C}$ & P & C \\
\hline Colorectal cancer & 50.6 & 44.7 & 4.2 & 7.5 & 0.5 & na & 55.3 & na \\
\hline Lung cancer & 20.3 & 30.9 & 0.9 & 0.9 & 0.3 & 0.9 & 21.5 & 32.7 \\
\hline Pancreatic duct cancer & 88.0 & 97.7 & 0.4 & na & nd & na & 88.6 & na \\
\hline Melanoma & 1.6 & 0.8 & 16.9 & 27.6 & 1.2 & 1.0 & 19.7 & 29.4 \\
\hline
\end{tabular}

The figures in P columns are derived from Prior et al. (9). Data on colon cancer and rectal cancer were fused. The same was done for lung adenocarcinoma and lung squamous cell carcinoma.

The figures in $\mathrm{C}$ columns originate from the paper by Cox et al. (21).

Both data sets originate from the same database (COSMIC) at different phases of its development.

nd - not detected; na - data not available

Table 2. Percentage distribution of the most common K-RAS mutations in colorectal cancer, lung cancer and pancreatic duct cancer $^{1}$

\begin{tabular}{|l|c|c|c|c|c|}
\hline \multirow{2}{*}{ Cancer } & \multicolumn{4}{|c|}{ K-RAS } \\
\cline { 2 - 6 } & G12C & G12D & G12V & G13D & All K-RAS $^{2}$ \\
\cline { 2 - 6 } & M/T & M/T & M/T & $18.1 / 6.0$ & $84.3 / 26.2$ \\
\hline Colorectal cancer & $9.7 / 3.2$ & $30.4 . / 10.1$ & $20.8 / 6.9$ & $2.1 / 0.4$ & $70.5 / 14.1$ \\
\hline Lung cancer & $35.5 / 7.1$ & $15.7 / 3.2$ & $17.1 / 3.4$ & $0.5 / 0.3$ & $83.3 / 50.4$ \\
\hline Pancreatic duct cancer & $3.0 / 1.8$ & $49.3 / 29.8$ & $30.5 / 18.5$ & M & \\
\hline
\end{tabular}

${ }^{1}$ The distribution shown copies the distribution of the individual RAS mutations as presented by Prior et al. (19). In that study the number of patients with individual K-RAS mutations were 2,344 for CRC, 2,197 for LC and 2,661 for PDC. The figures reporting individual K-RAS mutations were converted into percentages representing either the proportions of individual K-RAS mutations out of all K-RAS mutations (M) or the proportions of the individual K-RAS mutations out of all samples tested (T). CRC included both colon and rectal carcinomas, LC included lung adenocarcinoma, bronchoalveolar adenocarcinoma, non-small cell carcinoma, and squamous cell carcinoma. An example for explanation: in the case of CRC, G12C mutation was detected in $9.7 \%$ of RAS-mutated CRC but in only $3.2 \%$ of all CRC cancers tested G12C cysteine substituted for glycine at codon 12

2"All" means the indicated four mutations

$\mathrm{M}-\% \mathrm{~K}-\mathrm{RAS}$ mutated cancers within all RAS mutated cancers

$\mathrm{T}-\% \mathrm{~K}-\mathrm{RAS}$ mutated cancers within all K-RAS mutated cancers

G - glycine; C - cysteine; D - aspartic acid; V - valine 
The situation in the case of MEL is different. N-RAS mutations are more frequent than K-RAS mutations and are primarily carried by codon 61 . Table 3 presents the respective data based on the data presented by Prior et al. (19), which were converted into percentages of all melanomas tested. Although the frequencies of both Q61K and Q61R among RAS mutated melanomas are quite high (more than 40\%) in N-RAS mutated MEL, their part in this cancer is not very high (Table 3 ). However, it will be shown below that combining the indicated findings with RAF proto-oncogene alterations will markedly increase the percentage of this cancer carrying well defined oncogenic mutations and changed antigenic make-up. It seems justifiable to include peptides carrying Q61K and Q61R into the vaccine.

The association of RAS mutations with haematological malignancies is also quite strong (28). Again, they are predominantly associated with N-RAS mutations, less frequently with K-RAS mutations. In both instances they are primarily located in codons 12,13 and 61. According to the recent data their frequency is highest in acute myeloid leukaemia (14\%) and myeloma multiplex (19\% N-RAS, 18\% K-RAS) (9). One can expect that, if the vaccination proposed is effective in CRC and other cancers selected, and the most frequent mutations involved in haematological malignancies are clearly identified, the prophylaxis of haematological cancer will be on the programme of the next anti-cancer vaccination campaigns.

So far, the reasons for the associations of RAS mutants with various cancers are not well understood. In addition to the tissue specific factors, it is likely that some other factors are involved. It has recently been shown that differently mutated proteins differ in a number of biological activities such as membrane targeting, GTP hydrolysis rate and allosteric regulation. It is also possible that the new epitopes are differently recognized by the immune system; however, no valid data supporting this suspicion are available at this writing.

\section{Occurrence of Carcinogenic Mutations in B-RAF, a Downstream Signalling Molecule to RAS}

Mutated RAF is another cell oncogene involved in a large proportion of human cancers. Its denotation is derived from the virus inducing rapidly accelerated fibrosarcoma (v-RAF). Of the orthologs of this murine gene discovered in the human genome, one designated B-RAF, which is coding for serine-threonine kinase, is mutated in a significant portion of human cancers (29-35). According to these and other reports, RAF mutations are associated with 10 to $15 \%$ of human cancers, it is less than RAS mutations.
Similar to RAS, also RAF is an essential component of EGFR-mediated MAPK pathway, which is involved in multiple cell functions. RAF protein acts as an intermediate between the membrane bound RAS and MEK/ERK components of the MAPK pathway (Fig. 1). B-RAF mutations lead to B-RAF activation resulting in constitutive downstream signal transmission both in the cytoplasm and the nucleus. This results in cell proliferation and prolonged survival.

The most frequent oncogenic B-RAF mutation is located in codon 600 (in earlier reports mistaken for codon 599), leading to substitution of valine for glutamic acid (V600E). This mutation has been detected in up to $90 \%$ of B-RAF-mutated cancers (32-48). Data on the presence of V600E mutation in these cancers are shown in Table 4. V600E mutation has been detected in $10-15 \%$ of CRC $(33-38)$ but has been rare in LC $(39,40)$ and PDC (41). However, approximately $40-60 \%$ of MEL (42-43) harbour this mutation. B-RAF mutations are also frequent in thyroid cancer $(44,45)$.

In some other common cancers, the V600E mutation is less frequent. B-RAF mutations are rare in cervical and endometrial

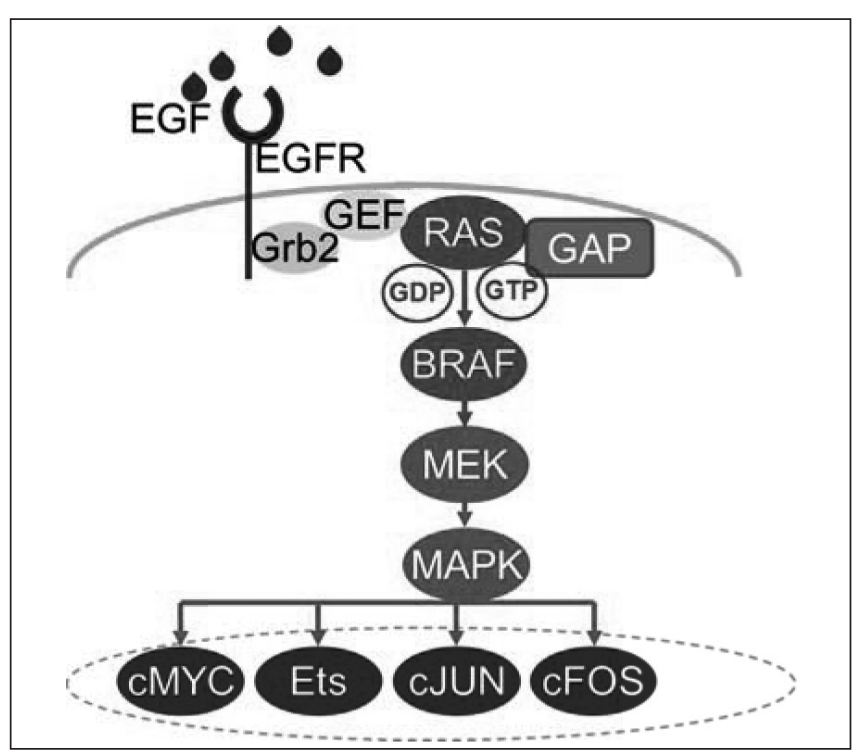

Fig. 1. RAS-BRAF signalling.

Epidermal growth factor (EGF) binds to the extracellular domain of the EGF receptor (EGFR). Activated EGFR recruits to its phosphorylated C-terminal cytoplasmic tail the growth factor receptor-bound protein 2 (Grb2) and a guanine nucleotide exchange factor (GEF), which exchanges GDP by GTP, activating RAF. The GTPase activating protein (GAP) stimulates the intrinsic GTPase activity of RAS. Active RAS dimerizes and binds RAF. Activated RAF activates mitogen-activated protein kinase (MAPK), kinase (MEK), which induces the final phosphorylation step of MAPK. Activated MAPK translocates into the nucleus where it activates transcription factors including c-Myc, Ets, c-Jun, and c-Fos.

Table 3. Percentage distribution of the most frequent N-RAS mutations in cutaneous melanomas before and after adjustment for the incidence of these mutations in all melanoma tests

\begin{tabular}{|l|c|c|c|}
\hline \multirow{3}{*}{ Cancer } & \multicolumn{3}{|c|}{ N-RAS } \\
\cline { 2 - 4 } & Q61K & Q61R & Both N-RAS \\
\cline { 2 - 4 } & M/T & M/T & $86.6 / 14.0$ \\
\hline Melanoma & $41.1 / 6.7$ & $46.3 / 7.3$ & \\
\hline
\end{tabular}

Calculated from the data presented by Prior et al. (19).

$\mathrm{M}-\% \mathrm{~N}-\mathrm{RAS}$ mutated melanomas within all $\mathrm{N}-\mathrm{RAS}$ mutated melanomas

$\mathrm{T}-\% \mathrm{~N}-\mathrm{RAS}$ mutated melanomas within all melanomas tested

$\mathrm{Q}$ - glutamine 
cancers (47) and are virtually absent in myeloid neoplasms (48). In general, they are rare in haematological cancers with a few exceptions such as the hairy cell leukaemia. In the study on a group of 47 patients suffering from this disease V600E mutation was detected in all of them (49). On the other hand, in the group of 180 multiple myeloma patients B-RAF mutations were detected in only 17 patients $(9 \%)$, of these only $41 \%$ carried V600E mutation (50). B-RAF mutations were detected in about $10 \%$ of brain tumors $(51)$.

B-RAF V600E mutation is quite common in some other rare carcinomas, such as craniopharyngioma (52) or Langerhans cell histiocytosis (53). Less clear is the situation in hepatocellular carcinoma (HC). In contrast with the earlier findings, which failed to demonstrate B-RAF mutations in HC (54), these mutations were detected in more than $20 \%$ patients in more recent studies $(55,56)$. It is possible that race factors played a role in these differences. It may be of interest that for some time it was held proven that B-RAF and RAS mutations were mutually exclusive, but more recently it has been demonstrated that exceptions exist. The low frequency of a simultaneous presence of both mutations may be due to the failure of the second event to provide an additional growth advantage to the already neoplastically transformed cells. Most studies have suggested that the tumours with B-RAF mutations (both CRC and MEL) possess worse prognosis than those in which malignancy has been associated with other molecular alterations. The reasons remain not to be completely understood. On the other hand, because of a new pharmacological armamentarium, the chemotherapy of tumors driven by B-RAF mutation has been more successful than those driven by mutated RAS (57).

\section{Mutations in RAS Oncogene-Induced Immune Re- sponses}

There is a growing evidence that cancer patients elicit immune reactions against the mutated RAS epitopes. The immune reactions develop either spontaneously in the course of the disease progression or are induced by ITC. The first findings along this line were already reported in the 1990s (57-65). Additional information and more details have been obtained in the last two decades (66-76).

The main message from those studies is that the respective cancer patients possessed $T$ cells capable of recognizing the respective neoepitopes and mounting immune responses against them. One can speculate that in the subjects who developed the cancer, their specifically sensitized $T$ cell pool had been either too small or possessed a too weak affinity to the target cells, making them incapable of eradicating the mutated (pre)cancerous cells.

Most of the results mentioned were obtained in ITC studies. The evidence that immunity against RAS mutation-associated cancers can be induced by specific prophylactic vaccines has repeatedly been obtained in animal systems. Immunization of C57 BL/6 mice with a K-RAS-mutated (G12S) 13-mer peptide induced immunity demonstrable after the challenge with cells derived from syngeneic bladder transitional cancer cells (cell line MB49) carrying the same RAS mutation (77). In our laboratory we used Syrian hamsters and a hamster cell line derived from non-oncogenic hamster embryonic fibroblasts (HEF cells), which had been co-transfected by plasmids carrying E6/ E7 genes of HPV16 and the human H-RAS gene harbouring the
G12V mutation (78). These cells were highly oncogenic for Syrian hamsters. Simultaneous immunization with both plasmids resulted in a significant protection against the challenge with the transformed cells. It is noteworthy that separate immunizations with either the E6/E7 plasmid or mutated H-RAS plasmid were also effective in this system. Since there is no mismatch between human and hamster H-RAS proteins (79), it is rather compelling that the effect observed was the result of an immune response to the neoepitope formed by the point mutation in codon 12 . Since Syrian hamsters are "semi-inbred", there was a possibility that a difference between the antigenic make-ups of the HEF cells and the animals used influenced the outcome of the test. We repeated the same experiments in the C57/BL6 mice using syngeneic MK III AB cells, which had been co-transformed by the HPV16 E6/E7 genes and activated H-RAS gene $(\mathrm{G} 12 \mathrm{~V})$. H-RAS proteins from humans and mice are homologous and thus the only difference between the wild type murine H-RAS protein and the mutated $\mathrm{H}-\mathrm{RAS}$ protein was the presence of G12V mutation. Although the immunization efficacy was of a lower degree than that observed in the hamster system, the treatment with the mutated H-RAS alone resulted in a significant delay in tumor formation $(p<0.03)$ comparable to that observed after immunization with the E6/E7 carrying plasmid carried out in parallel (80).

Possibly the most interesting results concerning the efficacy of the monoectopic anti-RAS prophylactic vaccines were obtained in mice exposed to a chemical carcinogen denoted DMBA (7,12-dimethylbenz(a)anthracene) and to TPA (tetradecanoylphorbol-acetate) acting as a promotor (81). DMBA induces skin cancers predominantly associated with mutation in the codon 61 of H-RAS (Q61L). Prior to the exposure to the carcinogen and TPA, the animals were immunized with either of the two vaccines. The first one was a DNA vaccine coding for the nonapeptide (amino acids No 59-67 of H-RAS protein) carrying the mutated epitope. The second one was prepared in dendritic XS106 cells, a Langerhans cell-derived cell line shown in previous experiments to exhibit a potent antigen presenting activity (82). XS106 cells were transfected with a lentivirus vector into which mutated HRAS oncogene had been subcloned. For the construction of both the vaccines a chimeric gene engineered to support MHC class I presentation of the neoepitope and selective expansion of CD8+ cells over regulatory CD4+ was used. The development of cancers after exposure to the carcinogen in the immunized animals was strongly reduced in the immunized animals. The rare tumors developed were either free of the mutated protein or expressed it in low amounts, they were smaller, grew slower and, in subpassages, their oncogenicity was reduced. This suggested that other mutations than Q61L were responsible for those tumors. Furthermore, the transfer of $\mathrm{T}$ cells from successfully immunized animals into mice bearing mutated H-RAS positive tumors resulted in their rapid regression. These data have shown that immunization against a single mutant epitope can protect against the development of carcinogen-induced tumors harbouring the same mutation.

While major support for the present concept of prophylactic anti-cancer vaccine development stems from experiments in a mice model, a strong backup for this concept has quite recently been provided by the demonstration of immune responses to mutated RAS epitopes in healthy subjects (83). This provides a strong support for the assumption that these healthy individuals experienced contact with cells expressing the respective RAS 
mutations earlier in their lives but have been capable of effectively controlling the neoplastic process.

\section{Immune Responses to Mutations in B-RAF Oncogene}

When compared with RAS mutations, much less is known about specific immune reactions against activated B-RAF. Still, monoclonal antibody against the V600E-mutated B-RAF is commercially available (Roche/Ventana) and is being extensively used in immunohistochemical detection of the mutation in diagnostic work, for prognosing the disease and for therapeutic and research purposes. The administration of several drugs targeted against mutated B-RAF is for a limited time-period quite efficient in the treatment of MEL associated with B-RAF mutations. This therapy is associated with a rather strong immune reaction against the cancer (84). The operative mechanisms are not fully understood. At least two explanations may be offered. First, the pharmacological therapy results in extensive tumor cell killing and the released tumor antigens induce a powerful immune response. Second, it is also possible that the drugs used reduce the production of immunosuppressive factors such as interleukin 6 (IL-6), IL-10 and vascular endothelial growth factor (VEGF). Increased production of these cytokines was shown to be associated with V600E mutation already more than a decade ago (84-86). It should be added that gaining resistance to B-RAF therapy is associated with the downregulation of the immune responses to MEL. These observations raise the question whether the targeted B-RAF pharmacotherapy is not in fact a form of immunotherapy (87).

Although it is likely that the mutated B-RAF epitope is involved in the immune reactivities following the targeted therapy, specific reactions against it have only rarely been reported. However, there are several observations suggesting that they are present in the cancer patients. Already more than a decade ago, it was reported that the cultivation of MEL patients' lymphocytes in the presence of 29-mer peptide encompassing the B-RAF mutation and the hypothetical MHC class II anchor residue, generated CD4+ cells, which were capable of recognizing both the mutated peptide and B-RAF mutation-positive MEL cells (88). Some patients lost the driving mutation during progression to metastatic disease (89). This phenomenon was most likely due to immune selection: anti-B-RAF immunity led to selection of cells with an oncogenic potential which, because of other mutations, became independent of the originally driving B-RAF mutation. This also suggests that the use of ITC vaccines carrying the mutated B-RAF epitope does not need to be an ideal means for immunotherapy of all advanced cancers. However, this should not limit the future use of mutated B-RAF for prophylactic purposes. Quite recently, identification and sequencing of $\mathrm{T}$ cell receptor (TCR) genes for mutated B-RAF were achieved. CD4+ cell line with the help of 20-mer peptide harbouring the V600E mutation from infiltrating lymphocytes was isolated. By its administration, stage IV MEL patient was cured (90).

RAS and BRAF are summarized in Table 5. They indicate that the heptavaccine proposed, if $100 \%$ effective, might prevent the development of CRC, PDC, MEL in a significant proportion of vaccinated subjects. It would be less effective in the case of LC.

\section{Prophylactic Vaccines vs. Cancer Immunotherapy}

There is no competition between these two modern approaches in the field of oncology. In spite of their different timing and targeting, both can be effective in reducing cancer mortality. Oncologists are fascinated by the success of vaccines preventing cancers of viral origin. This has been rendered possible because the cause of the respective cancers has been identified. It is the basis of the present concept for developing prophylactic cancer vaccines that the mutations of at least some proto-oncogenes are in the same logical category, i.e., that they may act as key causal factors. One can hope that such preparations will be extensively tested and hopefully introduced into medical practice in a not very distant future.

The aim of the immunotherapeutic approaches is to step up the efficacy of the classical cancer treatments, i.e., surgery, radiation and chemotherapy, with the aim to induce long-term cancer-free remission or, ideally, to cure the disease. At the present time, in spite of tremendous efforts and first significant successes of ITC, we are still at the start of the introduction of this medical modality. There is no doubt that more sophisticated methods with an increased efficacy will gradually be introduced in the years to come. The methodological progresses, e.g., the introduction of the next generation sequencing, analysis of liquid biopsies or the in silico predictions will enable personalized immunotherapeutic approaches to the treatment of patients. The recent recognition of the possibility to regulate the cytocidal interaction between $\mathrm{T}$ cells and cancer cells by bispecific low molecular adapters (91) should increase both ITC safety and efficacy. The demonstration that it is possible to engineer antibodies to make them to act as intrabodies (92) and the use of nanobodies (93) are gradually developing into other efficient ITC means. A great advantage of ITC is that the evaluation of its clinical effects can be achieved in a relatively short time, usually within months. Strong support should definitely be given to the research in this field, but this should not reduce efforts to develop prophylactic cancer vaccines.

The interaction between the immune system and cancer is a complex process, which is not yet fully understood. The authors of this paper belong to those who consider the cancer develop-

Table 4. Percentage of B-RAF Q6E mutations in selected human cancers

\begin{tabular}{|l|c|c|}
\hline Cancer & B-RAF V600E & References \\
\hline Colorectal cancer & $10-15$ & $33-38$ \\
\hline Lung cancer & 1.5 & 39,40 \\
\hline Pancreatic duct cancer & $<0.1$ & 41 \\
\hline Melanoma & $40-60$ & 42,43 \\
\hline Thyroid papillary cancer & $32-77$ & 44,45 \\
\hline
\end{tabular}


Table 5. Frequency of seven RAS and B-RAF mutations in four common human cancers - summary ${ }^{1,2}$

\begin{tabular}{|c|c|c|c|c|}
\hline \multirow{2}{*}{ Mutation } & \multicolumn{4}{|c|}{ Cancer } \\
\hline & Colorectal cancer & Lung cancer & Pancreatic duct cancer & Melanoma \\
\hline \multicolumn{5}{|l|}{ K-RAS } \\
\hline $\mathrm{G} 12 \mathrm{C}$ & 3.2 & 7.1 & 1.8 & nd \\
\hline G12D & 10.1 & 3.2 & 29.8 & 0.1 \\
\hline G12V & 6.9 & 3.4 & 18.5 & 0.2 \\
\hline G13D & 6.0 & 0.4 & 0.3 & 0.1 \\
\hline \multicolumn{5}{|l|}{ N-RAS } \\
\hline Q61K & & & & 6.7 \\
\hline Q61R & & & & 7.3 \\
\hline \multicolumn{5}{|l|}{ B-RAF } \\
\hline V600E & $10-15$ & & 1.5 & $40-60$ \\
\hline Total $^{3}$ & $36-41$ & 14 & 52 & $54-74$ \\
\hline
\end{tabular}

$1 \%$ of cancers with indicated mutation

${ }^{2}$ Data derived from reviews summarizing a large numbers of studies

${ }^{3}$ Numbers are rounded

nd - not detected

ment to be a consequence of the failure of the immune system to control the unlimited growth of the neoplastic cells. It is now broadly accepted that cancer-immune system interactions consist of three phases, denoted as the "3Es", namely Elimination, Equilibrium and Escape (94). Elimination is the proven victory of the immune system over the arising neoplasm. It is likely that it occurs many times during the life course of each individual. Equilibrium phase is the stage where the two processes, i.e., the immune reactivity and the (pre)neoplastic cells in a subclinical form coexist in the body because they are well-balanced. This irresolute state can last for long periods of time without being clinically recognized. The third phase, Escape, is characterized by the unlimited tumour growth due to additional mutations, which enhance the aggressivity of the transformed cells, or lead to the loss of control over the neoplastic process due to weakening immune reactivity. This is the time for starting therapy including ITC. The first two phases could be markedly influenced by prophylactic vaccines which, in fact, in the Equilibrium phase act as ITC. The scenarios which might be expected to occur after vaccination are as follows. First, due to inducing specific immunity, the Elimination of neoplastic or preneoplastic cells will be more effective and more frequent. Second, Equilibrium can be kept indefinitely or regress to Elimination. Both are beneficial.

A great disadvantage of the prophylactic cancer vaccines is the lengthy period needed for the corroboration of their efficacy, i.e., the reduction of cancer morbidity and mortality in the general population. This may take decades. Scientists involved in developing and administering vaccines for prevention of CRC and other cancers sharing the same mutations will most likely not be active in the time when the vaccine efficacy or its inefficacy will be determined. On the other hand, due to the recent progress in the tumor immunology and biology, laboratory tests can monitor the state of specific immunity to the cancer neoantigens, which develop following the vaccination. Qualified predictions on the vaccine efficacy are thereby possible. Similar tests are also capable of pursuing the persistence of immunity and to signal when revaccination is needed.

\section{DISCUSSION}

\section{Verification or Falsification}

In the first part of this paper a strategy was proposed, which might enable development of vaccines capable of preventing a significant proportion of human cancers. There are many ways how to examine the present hypothesis. Some of them are envisaged and discussed in the subsequent text.

\section{How to Interpret Occurrence of RAS and RAF Muta- tions in Human Cancers in Relationship to Prophy- lactic Vaccines Development}

The vaccines based on the mutated RAS or B-RAF epitopes, if sufficiently immunogenic, should be able to reduce the incidence of a number of human cancers. Before presenting the next section, it is necessary to make a short statement. The data shown in the previous sections (Tables 1-5) are not and cannot be sufficiently precise and reliable. They were collected from various studies, the results of which often markedly differed. There are several reasons for the differences encountered. Let us mention the most important ones: (a) the data on the mutation rates in different cancers were mostly obtained in observational studies of different size and design. The biggest inconsistencies were detected among small scale studies covering less than 100 patients, sometimes less than 30 patients. However, marked discrepancies are also apparent among reviews summarizing findings obtained in thousands of cases. (b) The undertakings were carried out in different populations. Racial differences were repeatedly reported. (c) The subjects tested were selected according to different criteria. (d) The materials analysed in various studies widely differed from samples obtained by fine needle biopsies to paraffine embedded tissue blocks. (e) Distinct methods either genetic or immunohistochemical were employed for the detection of the respective mutants. (f) In addition, the calculation and thus the figures presented in the big reviews were confounded by two factors. First, the rare cancers form- 
ing less than $3 \%$ of all human malignancies represented $20 \%$ of the tumors analysed by Prior et al. $(9,19)$ most likely due to an increased interest of both the clinicians and the pathologists in the molecular profiles of these cancers. Second, at least $13 \%$ of cancers are of infectious origin (1-4). For example, in cervical cancer which is caused by human papillomaviruses (HPV), the occurrence of RAS mutations was low and similar for all three isomers. The absence of any marked preference is at variance with the four cancers listed and suggests that neither RAS nor B-RAF mutations represent a decisive driving force in that malignancy.

In spite of these weaknesses the results obtained in the past independent studies are of a great importance. At least in the case of some cancers, the findings reported are not dissimilar. Fortunately enough, these include some of the most common cancers, which should be the primary targets of the immunization campaigns.

At this moment it is not known how immunogenic will be the heptavaccine proposed. As suggested below, it would be useful to test a few less-complex vaccines in the first round of vaccination and to compare their immunogenicity in parallel with the heptavaccine using a fitting animal model.

\section{Some Reflections and Speculations}

The vaccines proposed are not aimed at eradicating any special cancer type but at markedly reducing the incidence of some of the most common human cancers. Let us emphasize three points.

The RAS mutations, which are involved in the pathogenesis of up to $30 \%$ of all human malignancies of non-infectious origin, result in the formation of neoepitopes which are capable of inducing immune responses. These mutations are unequally distributed among different cancers including some of those which are the most frequent. The first part of our paper has presented data suggesting that immunization against the newly formed epitopes might result in the prevention of a large proportion of the respective cancers. If the proposed vaccines will work in the case of CRC and the other presently selected cancers, it is likely that their administration will also result in a marked reduction of some other cancers that share the same mutations, without respect whether K-RAS or N-RAS or H-RAS were involved in the transformation process $(9,19,20)$. Many of these cancers are of relatively low incidence, however. Therefore, in their case it would be difficult to determine the vaccination efficacy due to the lack of statistical power.

The molecular pathogenesis of cancers originating in the same tissues is not uniform. This creates an inevitable problem for the present type of prophylactic vaccines. Thus, in the vaccinated subjects the "mutated" RAS-B-RAF vaccines could act as a preventative means in a significant proportion of the cancer selected and also some other cancers provided that the vaccine is fully effective. However, the vaccine cannot work in this way in cancers in the development of which other driver mutations are involved. It will also be without any effect in preventing malignancies occurring as a result of the amplification of the wild type RAS proteins. It will also be of limited (if any) efficacy in preventing cancers in which the RAS (or B-RAF) mutation does not set in as a primary oncogenic event but as a consequence of genetic instability of the cells transformed due to other mutations. At this writing it is not known how many RAS- and B-RAFmutant positive cancers are afflicted in this way.
Let us assume that the heptavaccine under consideration is hundred percent effective (which certainly will not be the case) and speculate what might be its impact on the incidence of malignant tumours. One can expect that there will be differences among different countries due to differences in both the incidence of the cancers selected and the mutations involved in their origin. The above discussed data were mostly obtained in the USA. Provided that the whole US population is vaccinated and the vaccine fully effective, the estimated yearly incidence of the selected cancers may decrease by more than 550,000 cases (and 240,000 deaths) based on the last data from the National Cancer Institute $(9,19,20)$.

\section{Further Research is Needed, Suggestions in Brief for Next Series of Experiments}

It is necessary to demonstrate in experimental animals that the immunization with the mutated epitopes will be sufficient for the induction of immunity strong enough to prevent cancer development. Some evidence along this line has been mentioned in the first section of this paper, however, it is necessary to extend it under more rigorous experimental condition.

This topic should be tested first. As mentioned above, six K-RAS mutations at codons 12, 13 and 61, and one mutation of B-RAF at codon 600 are involved in the development of a large proportion of a variety of cancers, although at various frequencies. It is possible that different mutations located at the same codon also differ in their immunogenicity. For example, the high incidence of G12D in CRC (and in multiple other cancers) may be associated with its lower immunogenicity, whatever the mechanism responsible for it might be. Optimally, the vaccine should embrace all seven mutations encountered, i.e., K-RAS mutations G12D, G12V, G12C, and G13D; N-RAS mutations Q61K and Q61R; and B-RAF V600E mutation. However, will the immune response to such heptavaccine be sufficient enough to cover the activity of all these mutants? It seems appropriate to test the problem in mice using vaccines (e.g., mixtures of mutant peptides but preferentially some types of nanovaccines) of different complexity. They should be administered with an adjuvant, known to be effective in mice and permitted for use in human beings. Before this, it will be necessary to establish by CRISPR/ Cas9 technology or by other means a battery of cell lines derived from the same mouse strain and to determine their oncogenic potential in the syngeneic animals.

One of the many experimental strategies, which might be applied is to test immunogenicity of several combined vaccines in parallel. One set of the vaccine combinations is presented in Table 6. Vaccine A targets all seven mutated epitopes, vaccine B six of them (omitting B-RAF), vaccine C only the four K-RAS mutated epitopes, and vaccine D only the B-RAF mutated epitope. The vaccines should contain equal amount of each component. One of the many possible immunization schedules is to administer three vaccine doses, three weeks and three months apart. The groups should be sufficiently large to permit both in vitro and subsequent in vivo testing of specific immunity to all the epitopes included. The respective materials for in vitro tests should be collected, e.g., (a) three weeks after the second dose, (b) three weeks after the third dose, and (c) three months after the third dose. The activation of T cells will be tested by cultivating them in vitro in the presence of individual corresponding peptides and 
Table 6. Prophylactic cancer vaccines - a few examples

\begin{tabular}{|l|l|}
\hline Vaccine & Composition \\
\hline A & K-RAS G12C + K-RAS G12D + K-RAS G12V + K-RAS G13D + N-RAS Q61K + N-RAS Q61R + B-RAF V600E \\
\hline B & K-RAS G12C + K-RAS G12D + K-RAS G12V + K-RAS G13G + N-RAS Q61K + N-RAS Q61R \\
\hline C & K-RAS G12C + K-RAS G12D + K-RAS G12V + KRAS G13D \\
\hline D & B-RAF V600E \\
\hline
\end{tabular}

measuring the production of INF-gamma, IL-2 and TNF-alfa. ELISPOT will be used for the detection of T memory cells. To monitor the persistence of immunity, additional tests along the same lines should be performed six months and one year after the third dose of the vaccines. The remaining animals from all groups should be challenged separately with the corresponding syngeneic tumor cell lines carrying the different RAS mutations and the B-RAF mutation, respectively, in the same time periods. It does not seem absolutely necessary to include all the mutated cell lines into this initial experiment; however, it would be desirable to do so. The challenge dose should be $100 \mathrm{TID}_{50}$ in all instances. Genome instability in the tumours, if formed, should be analysed by single-cell DNA and RNA sequencing and/or immunohistochemistry. This experiment, if properly performed, should provide highly important information on the immunogenicity of the individual mutant epitopes, when applied in mixtures, as well as on the pathogenesis of the respective tumors on the molecular level. Sera from the animals will be tested for the presence of antibodies to all seven mutated epitopes.

It would be highly useful to perform one more study prior to the start of phase I study. This undertaking should be aimed at examining the validity of the " $3 E s "(94)$ hypothesis. To the best of our knowledge, the presence and distribution of the immune reactivities to the mutated RAS and RAF oncoproteins in a general population has not yet been reported, although the presence of immune responses to mutated RAS epitopes has been demonstrated (83). We therefore propose to select groups of ten to twenty unvaccinated healthy volunteers belonging to different age groups (21-30, 31-40, 41-50, 51-60, and so on) for such an investigation. Heavy smokers should form half of each age group, as smoking is known to induce RAS mutations. For additional control purposes, materials from patients with RAS and RAF mutations should be tested in parallel. The results may greatly contribute to defining the prevalence of anti-cancer immunity in the general population and thus to increase understanding of cancer epidemiology. In the case that more than one institute will be involved in such a trial, it would be highly useful to examine in parallel differently prepared vaccines and different vaccination schedules. The development and persistence of specific humoral and cell-mediated immunity should be tested in vitro. Recently, circulating cell-free (cfDNA) has received a lot of attention as a minimally invasive cancer biomarker for its ability to monitor disease progression, predict tumor recurrence and reflect the genetic heterogeneity of RAS mutations in the case of CRC and other cancers (95-97). As a noninvasive complement to traditional tissue biopsies, liquid biopsies detect and track cancer driver mutations from biofluids. Absence of the circulating mutated RAS from plasma of vaccinated DNA individuals in a longitudinal study could be used as a marker of immunogenicity of the vaccine in contrast to emerging mutations in plasma of non-vaccinated subjects.

\section{Sublata Causa, Tollitur Effectus}

It is the principal idea of the project outlined above that mutations of some proto-oncogenes, namely RAS and RAF, act as the key causal factors (CF) in many types of human cancers. However, different types of evidence for this hypothesis, as outlined above, widely differ in their significance. The experimental evidence obtained in animals has clearly indicated that the administration of the mutated oncogenes (in a variety of forms) precedes the development of cancer. In human cancer patients both the events, i.e., oncogenic action and the development of cancer, occurred in the past and it is not clear what came first. Were the discussed mutations induced by the chemical or physical carcinogens and did they serve as initial drivers of the oncogenic process, or was their presence in cancer cells a consequence of the well-known genetic instability of the cancer cells? The ideal way to clarify this point would be through a prospective study. However, this type of research, which is so fruitful in infectiology, is powerless within the present system. Means are not available to monitor a pathologic process that in most instances starts in one mutated cell.

In his deliberations on causality in medicine M. Susser used the philosophical terms "necessary" and "sufficient" to demonstrate four types of relationship between the putative causative factor (CF) and the disease (D) (98). They are presented in Table 7. It seems more than convincing that, from what we know on the relationship of RAS and RAF mutants with human cancer, cancer development corresponds to scheme number "4" claiming that the suspected causal factor is neither necessary nor sufficient. For example, nearly half of CRCs, do not harbour the described RAS or RAF mutations, suggesting that other genetic events were responsible for the development of those cancers.

For several decades criteria introduced by Hill (99) have been utilized for establishing the causal relationships in medicine. Their formulation was enforced by the situation in clinical virology in the 1950s. After wide-scale introduction of tissue cultures into the diagnostic work, more than one-hundred new viruses were isolated in the course of a few years and it was necessary to clarify their role, if any, in human disease. Hill's criteria included nine, partially overlapping, indicators presented in Table 8 . There is no doubt that their fulfilment markedly supports the probability of causal relation and, if the denotations of the criteria are semantically somewhat adjusted, it would become evident that most of them obey the rules of deductive logic. In our case, the in vitro demonstration of immune reactions specific for cancer neoantigens in vaccinated subjects will add an additional support for the essential role of the described mutations in the oncogenic process. However, there are two major difficulties preventing the use of Hill's criteria in full since the mutations under discussion are connected with different forms of cancer and there is a lack of specificity. The other problem is created by the length of the incubation period between the generation of the muta- 
Table 7. Logical schemes of relationship between causative factor and cancer

\begin{tabular}{|c|}
\hline CF is necessary and sufficient for inducing $C$ \\
\hline CF $\rightarrow C$ \\
\hline $\mathrm{CF}$ is necessary but is not sufficient for inducing $\mathrm{C}$ \\
\hline CF + cooperating factors -------- $\rightarrow C$ \\
\hline $\mathrm{CF}$ is not necessary but is sufficient for inducing $\mathrm{C}$ \\
\hline CF1 \\
\hline CF2 - $\rightarrow C$ \\
\hline CF3 $\rightarrow C$ \\
\hline $\mathrm{CF}$ is neither necessary nor sufficient for inducing $\mathrm{C}$ \\
\hline $\mathrm{CF}+$ cofactor $_{1+n}-\cdots-\cdots \mathrm{C}$ \\
\hline CF2 + cofactor $_{1+n}-\cdots$ \\
\hline CF3 + cofactor $_{1+n}-\cdots \rightarrow C$ \\
\hline
\end{tabular}

$\mathrm{CF}$ - causative factor; $\mathrm{C}$ - cancer

M. Susser, 1973; modified (98)

Table 8. Criteria for establishing causal relationship in medicine

\begin{tabular}{|l|}
\hline Strength of association \\
\hline Inconsistency (i.e. reproducibility) \\
\hline Specificity \\
\hline Temporal sequence \\
\hline Biological gradient \\
\hline Plausibility (i.e. epidemiological credibility) \\
\hline Coherence (i.e. biological credibility) \\
\hline Experimental evidence \\
\hline Analogy \\
\hline A. B. Hill, 1965; modified (99)
\end{tabular}

tion and the development of cancer, which may last for several decades. This opens the scene for the intervention of numerous, not clearly defined factors that may be hard to monitor. This is further complicated by the fact that in many chronic diseases including cancer it is difficult, if not impossible, to determine the time when the disease has started. Thus, what remains are the experimental evidence and analogy. However, these are more efficient for the generation of a new aetiological hypothesis than for its verification.

It seems that there is no other way than to go somewhat back when looking for decisive means to verify or falsify the hypothesis that RAS and B-RAF mutations are the events initiating the oncogenic process. The great 19 th century French physiologist and philosopher of science Claude Bernard formulated his attitude to the causality problem by his ingenious, but until recently nearly forgotten statement, "The only proof that one phenomenon is the cause of another is that by removing the first we stop the other" (100). It may be of interest that this is a paraphrase of a statement articulated 600 years earlier by Thomas Aquinas, one of the most eminent philosophers of the Middle Ages: "Sublata causa, tolliturs effectus" $(100,101)$. Since St. Thomas was the best expert in the work of Aristoteles and its interpreter, it is possible that his dictum was inspired by his great ancient predecessor.

Nevertheless, if the administration of the proposed anti-cancer vaccines will result in a significant decrease of cancers carrying the described mutations, the hypothesis that they are the causes of the respective cancers, will be convincingly verified. Yet, it is very well possible that the vaccination effect will be limited to certain mutations or to certain cancers. Whatever might be the outcome of vaccine administration there is a chance that it will significantly contribute to our understanding of the oncogenic process.

\section{CONCLUSIONS}

The strategy for cancer prevention proposed in this paper may seem innovative; however, it may also be considered as a logical outcome of the knowledge on cancer biology and immunology gained in the last two or three decades. Yet, at present it is a hypothesis. As such, it will either be verified or falsified in the future. Even if the vaccination will be found beneficial, full-size performance should not be expected. It is more likely that the results obtained in the future will invite substantial modification of the concept. There is little doubt that the mutated epitopes will induce immune reactions in the vaccinated subjects. The crucial points are how strong this immunity will be and how long it will persist. These are topics which have to be carefully studied. Depending on the outcome of those future studies, the optimal anti-cancer vaccination strategy will be developed and executed. RAS and B-RAF are not the only oncogenes which might be effective for the purpose. There are other candidate genes in the genome the products of which may be recognized as oncodrivers and used for the development of the next generations of the prophylactic vaccines.

As stressed in the first part of this communication the immunoprophylaxis of malignancies will not reduce the importance of cancer immunotherapy, radiotherapy, chemotherapy, and chemoprevention. In the years to come they will be systematically improved. These modifications will result in increasing their safety and efficacy. If the prophylactic anti-cancer vaccination results in highly significant decrease of the most frequent cancers, the other therapeutic modalities will gradually be limited to the rare cancers which will not be covered by the prophylactic vaccines used.

If these optimistic predictions materialize, it is possible to hope that to die of cancer will be a rather infrequent event at the end of this century.

\section{Conflict of Interests}

None declared

\section{REFERENCES}

1. Vonka V, Hamsíková E. Vaccines against human papillomaviruses--a major breakthrough in cancer prevention. Cent Eur J Public Health. 2007;15(4):131-9.

2. Pol S. Hepatitis: HBV vaccine-the first vaccine to prevent cancer. Nat Rev Gastroenterol Hepatol. 2015;12(4):190-1.

3. Lehours P, Ferrero RL. Review: Helicobacter: inflammation, immunology, and vaccines. Helicobacter. 2019;24 Suppl 1:e12644. doi: 10.1111/ hel. 12644.

4. Crews DW, Dombroski JA, King MR. Prophylactic cancer vaccines engineered to elicit specific adaptive immune response. Front Oncol. 2021;11:626463. doi: 10.3389/fonc.2021.626463.

5. Beckwith DM, Cudic M. Tumor-associated O-glycans of MUC1: Carriers of the glyco-code and targets for cancer vaccine design. Semin Immunol. 2020;47:101389. doi: 10.1016/j.smim.2020.101389. 
6. Chen W, Zhang Z, Zhang S, Zhu P, Ko JK, Yung KK. MUC1: structure, function, and clinic application in epithelial cancers. Int J Mol Sci. 2021;22(12):6567. doi: 10.3390/ijms22126567.

7. Finn OJ. Vaccines for cancer prevention: a practical and feasible approach to the cancer epidemic. Cancer Immunol Res. 2014;2(8):708-13.

8. Fletcher R, Wang YJ, Schoen RE, Finn OJ, Yu J, Zhang L. Colorectal cancer prevention: immune modulation taking the stage. Biochim Biophys Acta Rev Cancer. 2018;1869(2):138-48.

9. Prior IA, Hood FE, Hartley JL. The frequency of Ras mutations in cancer. Cancer Res. 2020;80(14):2969-74

10. Bos JL. ras oncogenes in human cancer: a review. Cancer Res. 1989 Sep 1;49(17):4682-9. Erratum in: Cancer Res 1990;50(4):1352.

11. Tsuchida N, Murugan AK, Grieco M. Kirsten Ras* oncogene: significance of its discovery in human cancer research. Oncotarget. 2016;7(29):4671733.

12. Hobbs GA, Der CJ, Rossman KL. RAS isoforms and mutations in cancer at a glance. J Cell Sci. 2016;129(7):1287-92.

13. Muñoz-Maldonado C, Zimmer Y, Medová M. A Comparative analysis of individual RAS mutations in cancer biology. Front Oncol. 2019;9:1088. doi: 10.3389/fonc. 2019.01088 .

14. Serebriiskii IG, Connelly C, Frampton G, Newberg J, Cooke M, Miller $\mathrm{V}$, et al. Comprehensive characterization of RAS mutants in colon and rectal cancer. Nat Commun. 2019;10: 3722. Doi: 10.1038/s41467-01911530-0.

15. Guo YJ, Pan WW, Liu SB, Shen ZF, Xu Y, Hu LL. ERK/MAPK signalling pathway and tumorigenesis. Exp Ther Med. 2020;19(3):1997-2007.

16. Haigis KM, Kendall KR, Wang Y, Cheung A, Haigis MC, Glickman JN, et al. Differential effects of oncogenic K-Ras and N-Ras on proliferation, differentiation and tumor progression in the colon. Nat Genet 2008;40(5):600-8.

17. Hartley ML, Bade NA, Prins PA, Ampie L, Marshall JL. Pancreatic cancer, treatment options, and GI-4000. Hum Vaccin Immunother. 2014;10(11):3347-53

18. Burd CE, Liu W, Huynh MV, Waqas MA, Gillahan JE, Clark KS, et al. Mutation-specific RAS oncogenicity explains NRAS codon 61 selection in melanoma. Cancer Discov. 2014;4(12):1418-29.

19. Prior IA, Lewis PD, Mattos C. A comprehensive survey of Ras mutations in cancer. Cancer Res. 2012;72(10):2457-67.

20. Prior IA. The disease burden of Ras [Internet]. Bethesda: National Cancer Institute; 2020 [cited 2021 Dec 3]. Available from: https://www. cancer.gov/research/key-initiatives/ras/ras-central/blog/2020/prior-rasin-cancer-databases.

21. Cox AD, Fesik SW, Kimmelman AC, Luo J, Der CJ. Drugging the undruggable Ras: mission possible? Nat Rev Drug Discov. 2014;13(11):828-51.

22. Jang EK, Song DE, Sim SY, Kwon H, Choi YM, Jeon MJ, et al. NRAS codon 61 mutation is associated with distant metastasis in patients with follicular thyroid carcinoma. Thyroid. 2014;24(8):1275-81.

23. Khan AQ, Kuttikrishnan S, Siveen KS, Prabhu KS, Shanmugakonar M, Al-Naemi HA, et al. RAS-mediated oncogenic signaling pathways in human malignancies. Semin Cancer Biol. 2019;54:1-13.

24. Gutiérrez-Castañeda LD, Nova JA, Tovar-Parra JD. Frequency of mutations in BRAF, NRAS, and KIT in different populations and histological subtypes of melanoma: a systemic review. Melanoma Res. 2020;30(1):62-70.

25. Pantsar T. The current understanding of KRAS protein structure and dynamics. Comput Struct Biotechnol J. 2019;18:189-98

26. Heppt MV, Siepmann T, Engel J, Schubert-Fritschle G, Eckel R, Mirlach $\mathrm{L}$, et al. Prognostic significance of BRAF and NRAS mutations in melanoma: a German study from routine care. BMC Cancer. 2017;17(1):536. doi: 10.1186/s12885-017-3529-5.

27. Hobbs GA, Der CJ. RAS Mutations Are Not Created Equal. Cancer Discov. 2019;9(6):696-8.

28. Ward AF, Braun BS, Shannon KM. Targeting oncogenic Ras signaling in hematologic malignancies. Blood. 2012;120(17):3397-406.

29. Davies H, Bignell GR, Cox C, Stephens P, Edkins S, Clegg S, et al. Mutations of the BRAF gene in human cancer. Nature. 2002;417(6892):949-54.

30. Seppälä TT, Böhm JP, Friman M, Lahtinen L, Väyrynen VM, Liipo TK, et al. Combination of microsatellite instability and BRAF mutation status for subtyping colorectal cancer. Br J Cancer. 2015;112(12):1966-75.

31. Pakneshan S, Salajegheh A, Smith RA, Lam AK. Clinicopathological relevance of BRAF mutations in human cancer. Pathology. 2013;45(4):346 56.

32. Zaman A, Wu W, Bivona TG. Targeting oncogenic BRAF: past present, and future. Cancers (Basel). 2019;11(8):1197. doi: 10.3390/ cancers 11081197
33. Barras D. BRAF Mutation in colorectal cancer: an update. Biomark Cancer. 2015;7(Suppl 1):9-12.

34. Ates $\mathrm{O}$, Yalcin S. Concomitant RAS and BRAF mutation in colorecta cancer - a report of 7 cases. Indian J Cancer. 2019;56(2):176-9.

35. Corcoran RB, André T, Atreya CE, Schellens JHM, Yoshino T, Bendell JC, et al. Combined BRAF, EGFR, and MEK inhibition in patients with BRAFV600E-mutant colorectal cancer. Cancer Discov. 2018;8(4):428-43.

36. Kopetz S, Grothey A, Yaeger R, Van Cutsem E, Desai J, Yoshino T, et al. Encorafenib, binimetinib, and cetuximab in BRAF V600E-mutated colorectal cancer. N Engl J Med. 2019;381(17):1632-43.

37. Dvorak K, Higgins A, Palting J, Cohen M, Brunhoeber P. Immunohistochemistry with anti-BRAF V600E (VE1) mouse monoclonal antibody is a sensitive method for detection of the BRAF V600E mutation in colon cancer: evaluation of 120 cases with and without KRAS mutation and literature review. Pathol Oncol Res. 2019;25(1):349-59.

38. Wu M, Kim YS, Ryu HS, Choi SC, Kim KY, Park WC, et al. MSI status is associated with distinct clinicopathological features in BRAF mutation colorectal cancer: A systematic review and meta-analysis. Pathol Res Pract. 2020;216(1):152791. doi: 10.1016/j.prp.2019.152791.

39. Tissot C, Couraud S, Tanguy R, Bringuier PP, Girard N, Souquet PJ. Clinical characteristics and outcome of patients with lung cancer harboring BRAF mutations. Lung Cancer. 2016;91:23-8.

40. Cui G, Liu D, Li W, Fu X, Liang Y, Li Y, et al. A meta-analysis of the association between BRAF mutation and nonsmall cell lung cancer. Medicine (Baltimore). 2017;96(14):e6552. doi: 10.1097/MD.0000000000006552.

41. Schultz NA, Roslind A, Christensen IJ, Horn T, Høgdall E, Pedersen LN et al. Frequencies and prognostic role of KRAS and BRAF mutations in patients with localized pancreatic and ampullary adenocarcinomas. Pancreas. 2012;41(5):759-66.

42. Cheng L, Lopez-Beltran A, Massari F, MacLennan GT, Montironi R. Molecular testing for BRAF mutations to inform melanoma treatment decisions: a move toward precision medicine. Mod Pathol. 2018;31(1):24 38.

43. Patel H, Yacoub N, Mishra R, White A, Long Y, Alanazi S, et al. Curren advances in the treatment of BRAF-mutant melanoma. Cancers (Basel). 2020;12(2):482. doi: 10.3390/cancers 12020482.

44. Xing M, Alzahrani AS, Carson KA, Viola D, Elisei R, Bendlova B, et al Association between BRAF V600E mutation and mortality in patients with papillary thyroid cancer. JAMA. 2013;309(14):1493-501.

45. Pyo JS, Sohn JH, Kang G. BRAF Immunohistochemistry using clone VE1 is strongly concordant with BRAF(V600E) mutation test in papillary thyroid carcinoma. Endocr Pathol. 2015;26(3):211-7.

46. Karbowniczek M, Henske EP. The role of tuberin in cellular differentiation: are B-Raf and MAPK involved? Ann N Y Acad Sci. 2005;1059:168-73.

47. Pappa KI, Choleza M, Markaki S, Giannikaki E, Kyroudi A, Vlachos G, et al. Consistent absence of BRAF mutations in cervical and endometria cancer despite KRAS mutation status. Gynecol Oncol. 2006;100(3):596600

48. Trifa AP, Popp RA, Cucuianu A, Coadă CA, Urian LG, Militaru MS, et al. Absence of BRAF V600E mutation in a cohort of 402 patients with various chronic and acute myeloid neoplasms. Leuk Lymphoma. 2012;53(12):2496-7

49. Tiacci E, Trifonov V, Schiavoni G, Holmes A, Kern W, Martelli $\mathrm{MP}$, et al. BRAF mutations in hairy-cell leukemia. N Engl J Med. 2011;364(24):2305-15

50. Xu J, Pfarr N, Endris V, Mai EK, Md Hanafiah NH, Lehners N, et al. Molecular signaling in multiple myeloma: association of RAS/RAF mutations and MEK/ERK pathway activation. Oncogenesis. 2017;6(5):e337. doi: 10.1038/oncsis.2017.36

51. Maraka S, Janku F. BRAF alterations in primary brain tumors. Discoy Med. 2018;26(141):51-60.

52. Brastianos PK, Taylor-Weiner A, Manley PE, Jones RT, Dias-Santagata D, Thorner AR, et al. Exome sequencing identifies BRAF mutations in papillary craniopharyngiomas. Nat Genet. 2014;46(2):161-5.

53. Badalian-Very G, Vergilio JA, Degar BK, MacConaill LE, Brandner B, Calicchio ML, et al. Recurrent BRAF mutations in Langerhans cell histiocytosis. Blood. 2010;116(11):1919-22.

54. Tannapfel A, Sommerer F, Benicke M, Katalinic A, Uhlmann D, Witzigmann $\mathrm{H}$, et al. Mutations of the BRAF gene in cholangiocarcinoma but not in hepatocellular carcinoma. Gut. 2003;52(5):706-12.

55. Colombino M, Sperlongano P, Izzo F, Tatangelo F, Botti G, Lombard A, et al. BRAF and PI3KCA genes are somatically mutated in hepatocellular carcinoma among patients from South Italy. Cell Death Dis. 2012;3(1):e259. doi: 10.1038/cddis.2011.136. 
56. Hou W, Liu J, Chen P, Wang H, Ye BC, Qiang F. Mutation analysis of key genes in RAS/RAF and PI3K/PTEN pathways in Chinese patients with hepatocellular carcinoma. Oncol Letts. 2014;8(3):1249-54.

57. Gedde-Dahl T 3rd, Spurkland A, Eriksen J, Thorsby E, Gaudernack G. Memory $\mathrm{T}$ cells of a patient with follicular thyroid carcinoma recognize peptides derived from mutated p21 ras (Gln-->Leu61). Int Immunol. 1992;4(11):1331-7.

58. Gedde-Dahl T 3rd, Eriksen JA, Thorsby, Gaudernack G. T-cell responses against products of oncogenes: generation and characterization of human T-cell clones specific for p21 ras-derived synthetic peptides. Hum Immunol. 1992;33(4):266-74.

59. Fossum B, Gedde-Dahl T 3rd, Hansen T, Eriksen JA, Thorsby E, Gaudernack G. Overlapping epitopes encompassing a point mutation (12 Gly-->Arg) in p21 ras can be recognized by HLA-DR, -DP and -DQ restricted T cells. Eur J Immunol. 1993;23(10):2687-91.

60. Cheever MA, Chen W, Disis M, Takahashi M, Peace DJ. T-cell immunity to oncogenic proteins including mutated ras and chimeric bcr-abl. Ann NY Acad Sci. 1993;690:101-12.

61. Fenton RG, Keller CJ, Hanna NN, Taub DD. Induction of T-cell immunity against Ras oncoproteins by soluble protein or Ras-expressing Escherichia coli. J Natl Cancer Inst. 1995;87(24):1853-61.

62. Gjertsen MK, Saeterdal I, Thorsby E, Gaudernack G. Characterisation of immune responses in pancreatic carcinoma patients after mutant $\mathrm{p} 21$ ras peptide vaccination. Br J Cancer. 1996;74(11):1828-33

63. Abrams SI, Hand PH, Tsang KY, Schlom J. Mutant ras epitopes as targets for cancer vaccines. Semin Oncol. 1996;23(1):118-34.

64. Gjertsen NK, Gaudemack G. Mutated ras peptides as vaccines in immunotherapy of cancer. Vox Sang. 1998;74 (Suppl 2):489-95.

65. Khleif SN, Abrams SI, Hamilton JM, Bergmann-Leitner E, Chen A, Bastian A, et al. A phase I vaccine trial with peptides reflecting ras oncogene mutations of solid tumors. J Immunother. 1999;22(2):155-65.

66. Hunger RE, Brand CU, Streit M, Eriksen JA, Gjertsen MK, Saeterdal I, et al. Successful induction of immune responses against mutant ras in melanoma patients using intradermal injection of peptides and GM-CSF as adjuvant. Exp Dermatol. 2001;10(3):161-7.

67. Linard B, Bezieau S, Benlalam H, Labarrière N, Guilloux Y, Diez E, et al. A ras-mutated peptide targeted by CTL infiltrating a human melanoma lesion. J Immunol. 2002;168(9):4802-8.

68. Carbone DP, Ciernik IF, Kelley MJ, Smith MC, Nadaf S, Kavanaugh $\mathrm{D}$, et al. Immunization with mutant p53- and K-ras-derived peptides in cancer patients: immune response and clinical outcome. J Clin Oncol. 2005;23(22):5099-107.

69. Kubuschok B, Neumann F, Breit R, Sester M, Schormann C, Wagner $\mathrm{C}$, et al. Naturally occurring T-cell response against mutated p21 ras oncoprotein in pancreatic cancer. Clin Cancer Res. 2006;12(4):1365-72.

70. Meyer RG, Korn S, Micke P, Becker K, Huber C, Wölfel T, et al. An open-label, prospective phase I/II study evaluating the immunogenicity and safety of a ras peptide vaccine plus GM-CSF in patients with nonsmall cell lung cancer. Lung Cancer. 2007;58(1):88-94.

71. Rahma OE, Hamilton JM, Wojtowicz W, Dakheel O, Bernstein S, Liewehr DJ, et al. The immunological and clinical effects of mutated ras peptide vaccine in combination with IL-2, GM-CSF, or both in patients with solid tumors. J Transl Med. 2014;12:55. doi: 10.1186/1479-5876-12-55.

72. Pandolfi F, Cianci R, Pagliari D, Casciano F, Bagalà C, Astone A, et al. The immune response to tumors as a tool toward immunotherapy. Clin Dev Immunol. 2011;2011:894704. doi: 10.1155/2011/894704.

73. Polidoro MA, Milane F, Soldani CV, Franceschini B, Anselmo A, Colombo FS, et al. Impact of RAS mutations on the immune infiltrate of colorectal liver metastases: a preliminary study. J Leukoc Biol. 2020;108(2):715-21.

74. Shahda S, O'Neil B. GI-4000 in KRAS mutant cancers. Expert Opin Investig Drugs. 2014;23(2):273-8.

75. Szcepaniak Sloane RA, Gopalakrishnan V, Reddy SM, Zhang X, Reuben A, Wargo JA. Interaction of molecular alterations with immune response in melanoma. Cancer. 2017;123(S11):2130-42.

76. Chen J, Xiao-Zhong G, Qi XS. Clinical outcomes of specific immunotherapy in advanced pancreatic cancer: a systematic review and meta-analysis. J Immunol Res. 2017;2017:8282391. doi: 10.1155/2017/8282391.

77. Luo Y, Chen X, Han R, Chorev M, Dewolf WC, O'Donnell MA. Mutated ras p21 as a target for cancer therapy in mouse transitional cell carcinoma. J Urol. 1999;162(4):1519-26.

78. Šmahel M, Sobotkova E, Vonka V, Hamsikova E, Zak R, Kitasato H, et al. DNA vaccine against oncogenic hamster cells transformed by HPV16 E6/E7 oncogenes and the activated ras oncogene. Oncol Rep. 1999;6(1):211-5.
79. Fenton RG, Taub DD, Kwak LW, Smith MR, Longo DL. Cytotoxic T-cell response and in vivo protection against tumor cells harboring activated ras proto-oncogenes. J Natl Cancer Inst. 1993;85(16):1294-302.

80. Šmahel M, Sobotková E, Bubenik J, Símová J, Zák R, Ludviková V, et al. Metastatic MHC class I-negative mouse cells derived by transformation with human papillomavirus type 16. Br J Cancer. 2001;84(3):374-80.

81. Nasti TH, Rudemiller KJ, Cochran JB, Kim HK, Tsuruta Y, Fineberg NS, et al. Immunoprevention of chemical carcinogenesis through early recognition of oncogene mutations. J Immunol. 2015;194(6):2683-95.

82. Timares L, Takashima A, Johnston SA. Quantitative analysis of the immunopotency of genetically transfected dendritic cells. Proc Natl Acad Sci U S A. 1998;95(22):13147-52.

83. Holmström MO, Andersen MH. Healthy donors harbor memory T cell responses to RAS neo-antigens. Cancers (Basel). 2020;12(10):3045. doi: 10.3390/cancers12103045.

84. LoRusso PM, Schalper K, Sosman J. Targeted therapy and immunotherapy: emerging biomarkers in metastatic melanoma. Pigment Cell Melanoma Res. 2020;33(3):390-402.

85. Ilieva KM, Correa I, Josephs DB, Karagiannis P, Egbuniwe IU, Cafferkey MJ, et al. Effects of BRAF mutations and BRAF inhibition on immune responses to melanoma. Mol Cancer Ther. 2014;13(12):2769-83.

86. Sharkey MS, Lizée G, Gonzales MI, Patel S, Topalian SL. CD4(+) T-cell recognition of mutated B-RAF in melanoma patients harboring the V599E mutation. Cancer Res. 2004;64(5):1595-9.

87. Kelley MC. Immune responses to BRAF-targeted therapy in melanoma: Is targeted therapy immunotherapy? Crit Rev Oncol. 2016;21(1-2):83-91.

88. Somasundaram P, Swoboda R, Caputo L, Otvos L, Weber B, Volpe P, et al. Human leukocyte antigen-A2-restricted CTL responses to mutated BRAF peptides in melanoma patients. Cancer Res. 2006;66(6):3287-93.

89. Andersen MH, Fensterle J, Ugurel S, Reker S, Houben R, Guldberg P, et al. Immunogenicity of constitutively active V599EBRAF. Cancer Res. 2004;64(15):5456-60.

90. Veatch JR, Lee SM, Fitzgibbon M, Chow IT, Jesernig B, Schmitt T, et al. Tumor-infiltrating BRAFV600E-specific CD4+ T cells correlated with complete clinical response in melanoma. J Clin Invest. 2018;128(4):1563-8.

91. Lu YJ, Chu H, Wheeler LW, Nelson M, Westrick E, Matthaei JF, et al. Preclinical evaluation of bispecific adaptor molecule controlled folate receptor CAR-T cell therapy with special focus on pediatric malignancies. Front Oncol. 2019;9:151. doi: 10.3389/fonc.2019.00151.

92. Marschall ALJ, Dubel S. Antibodies inside of a cell can change its outside: can intrabodies provide a new therapeutic paradigm? Comput Struct Biotechnol J. 2016;14:304-8.

93. Jovčevska I, Muyldermans S. The therapeutic potential of nanobodies. BioDrugs. 2020;34(1):11-26.

94. Dunn GP, Old LJ, Schreiber RD. The three Es of cancer immuno-editing. Ann Rev Immunol. 2004;22:329-60.

95. Klein-Scory S, Wahner I, Maslova M, Al-Sewaidi Y, Pohl M, Mika $\mathrm{T}$, et al. Evolution of RAS mutational status in liquid biopsies during first-line chemotherapy for metastatic colorectal cancer. Front Oncol. 2020;10:1115. doi: 10.3389/fonc.2020.01115.

96. Germetaki T, Nicholls C, Adams RA, Braun M, Rogan J, Moghadam S, et al. Blood-based RAS mutation testing: concordance with tissue-based RAS testing and mutational changes on progression. Future Oncol. 2020;16(28):2177-89.

97. Zvereva M, Roberti G, Durand G, Voegele C, Nguyen MD, Delhomme TM, et al. Circulating tumour-derived KRAS mutations in pancreatic cancer cases are predominantly carried by very short fragments of cell-free DNA. EBioMedicine. 2020;55:102462. doi: 10.1016/j. ebiom.2019.09.042.

98. Susser M. Causal thinking in the health sciences: concepts and strategies of epidemiology. New Yor: Oxford University Press; 1973.

99. Hill AB. The environment and disease: association or causation? Proc R Soc Med. 1965;58(5):295-300.

100. Vonka V. Causality in medicine: the case of tumours and viruses. Philos Trans R Soc Lond B Biol Sci. 2000;355:1831-41.

101. Gillies D. Indeterministic causality. In: Gillies D. Causality, probability, and medicine. Abingdon: Routledge; 2019. 195-203.

Received December 1, 2021

Accepted in revised form December 23, 2021 